\title{
Chemical Sensing via Single Input - Multi Output Approach
}

\author{
Jorel C., Robbes D., Radu R. C., Denoual M. \\ GREYC \\ UMR 6072 (Unicaen, Ensicaen, CNRS) \\ Caen, France \\ corentin.jorel@unicaen.fr
}

\author{
Grand J., Bazin P., Mintova S. \\ LCS \\ ENSICAEN \\ Caen, France
}

\begin{abstract}
The concept of single input - multi output (SIMO) sensors is investigated for the purpose of chemical sensing and more precisely gas sensing. The measurement of a single gas is coupled to different outputs, each involving various transduction mechanisms. The concept is especially matched to gas sensing via selective adsorbing mechanism in an active layer, the mass or the electrical polarization of which both depend on the adsorbed amount. In this work, adsorbing layers are deposited on pairs of light membranes, each equipped with a meander shaped, resistive and temperature dependent layer. The latter provides heater - thermometer functions while the balanced pair assembly also makes them well matched to capacitive transduction and reading. This paper reports on such systems built either with industrial Mylar membranes or with Silicon based membranes covered with zeolite layers to introduce selectivity.
\end{abstract}

Keywords-sensor; SIMO; capacitor sensor; thermometer sensor

\section{INTRODUCTION}

The single input - multiple output sensing concept, referred here after as SIMO, starts from that short analysis of the common behavioral of sensors: despite it often involves a cascade of intermediary transformations between the input physical quantity (measurand) and the sensor output, it is making good use of only one outgoing physical quantity. While in systems using the SIMO concept, a primary transformation of the measurand signal must be operated in its single test body, but parallel or sequential secondary transformations are allowed and/or switchable between this single test body and some secondary subsystems. The latter are able to produce analog images of the measurand variations that have experienced different physical transformations through the subsystems. That SIMO concept was investigated earlier to improve dynamics and frequency range of three axis magnetometers [1]. We report on its possible interests in the domain of chemical sensing.

The paper is arranged as follows. Section II details the SIMO concept, and discusses why the domain of chemical sensing is particularly attractive to be implemented. Section III is dedicated to the various experimental facets of our works, including two technological ways, both using membranes and an active and selective adsorbing layer. In addition, the controlled analyte application to the devices is presented. Section IV presents our results and discussion.

\section{Single INPUT - Multi OUTPUT CONCEPT}

\section{A. Generalities}

Primarily, the SIMO is supposed to be applied to sensing devices where the measurand, although easy to define, is not so conveniently returned from the single outgoing quantity associated to the measuring principle involved in the device. Such a situation arises in many circumstances due to, say, the weakness of the measuring principle used, the strength of various noise sources and effects, and most of all, unavoidable influence effects from other physical quantities, and, above all, temperature effects. On the other hand, modern electronics and signal processing made available very large dynamic ranges and bandwidths, real time and advanced signal processing. Along the same timescale, powerful tools to design microsystems and technological processes to fabricate them have been developed and adopted by the community. It induces that advanced microsystems now may include on the same chip more components that belong to different class of physical problems. All these considerations led us to the development of the SIMO concept. The measurand enters a common test body under the effect of a primary physical mechanism, or a set of physical mechanism, provided that the entered form of the measurand is common to the set of outgoings that are, afterwards, interacting with the test body. The latter are making good use of different transducing mechanisms applied to the stored form of the measurand. These different transducing mechanisms then produce multiples outgoing of SIMO based sensing devices, the redundancy/difference of which are to be used to improve the measuring process (influences identifications and removing, noise identification and removing, ...).

\section{B. Specificities}

Chemical species, or analytes, either in liquid or vapour phase, fall in the class of well specified measurands: a well defined chemical composition and well defined chemical reactivity and physical properties. But, except in the case a mass spectroscopy/chromatography based systems, most of the time, the analytes are present in mixtures and not as single component. Moreover, the interesting compounds to be sensed exist in low concentrations. It follows that a particular chemical species is a measurand that needs both a selective detecting system (discrimination of that particular species among the

C2-STM project has been funded by the CNRS program DEFIinstrumentations aux limites. 
others in the mixture), a good detectivity (how low is the detecting level?) and a large dynamic or measuring range (how much of that particular species is to be managed?). Such specifications of a measuring system are not easily fulfilled. As an example, one can quickly analyze those sensor based on selective adsorbing layer deposited on Quartz Crystals Microbalance (QCM), because the mass transduction makes good use of the crystal piezoelectric effect. The functional signal path from the measurand form (analyte concentration in a vector gas) to the outgoing physical quantity (frequency deviation of an AC voltage signal) is associated with a mechanical resonance shift due to the amount of adsorbed mass, while the biasing use the piezoelectric effect and reading is of capacitive nature. On the other hand, a SIMO based device cannot be described straightforwardly, due to the set of biasing and reading modes.

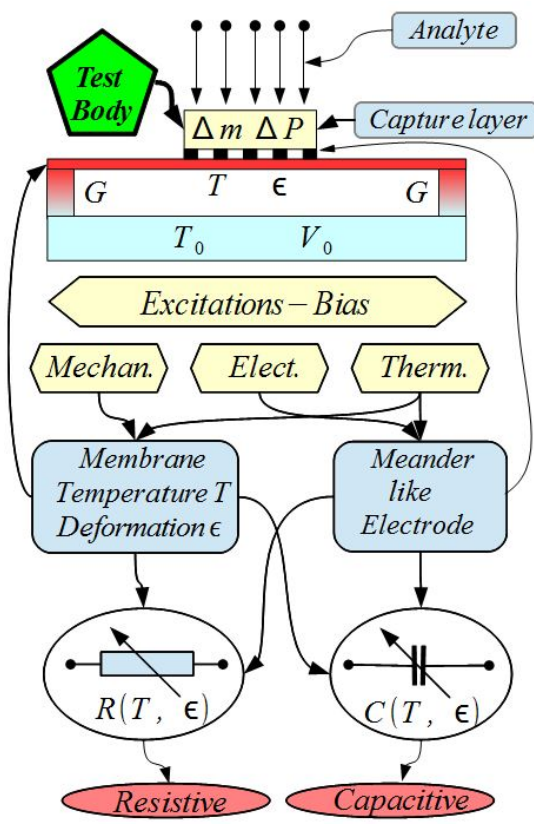

Fig. 1. Functional drawing of the proposed SIMO system. Letters $m$ and $P$ abbreviate mass and electrical polarizability, while $T$ and $\varepsilon$, temperature and deformation, and $V_{0}$ the reference voltage and $G$ the conductance of thermal leak from the membrane to the heat reservoir at $T_{0}$.

A functional drawing is useful to do this, and is proposed in Fig.1. It uses the same adsorbing layer as the ones deposited on QCMs. But several signal paths are proposed. A visual inspection of Fig. 1 highlights both the single test body which is needed in SIMO systems, together with the MEMS structure that allows a few biasing modes and/or reading modes. It uses a meander like patterned metallic layer which can be used to implement either a bolometric/calorimetric measuring principle with heating and temperature reading/control [2], or to implement mechanical deformation reading because of the strain gauge effects whenever the membrane is worked in a vibration mode. The same metallic and patterned meander can also be used as a simple conducting electrode, which then allows capacitive readings, either of the mechanical deformation or of the polarizability variation in the vicinity of the dielectric layer that surrounds the electrode.
In the case of chemical sensing that primarily converts the searched chemical species in a mass which can be directly read through a frequency shift of the test-body/membrane mechanical resonance, or mirrored through a heat capacity variation which would be identified using a bolometric/calorimetric approach. The frequency shift can be read using resistive mode or capacitive mode. Finally, if the adsorbed single chemical species have some electrical dipole moment, the associated polarizability variation in the vicinity of the meander electrode is as well related to the amount of analytes adsorbed.

\section{EXPERIMENTAL DETAILS}

\section{A. Samples preparation}

Two fabrication processes were selected to prepare suspended membranes with zeolite thin films used as the selective adsorbing layers. The zeolites are nano- or microsized porous inorganic crystals, which contain molecular sized regular channels (pores). Because of their unique pore size and shape, their large surface areas, and various chemical compositions, the zeolites can discriminate between molecules based on size and shape selectivity. In addition to the classical applications in sorption and catalysis, zeolites are also attractive for advanced technologies including highly selective separation membranes, host guest systems, and chemical sensors [2]. As a sensing layer, zeolites are very favorable due to their extremely high thermal stability and chemical resistance [3].

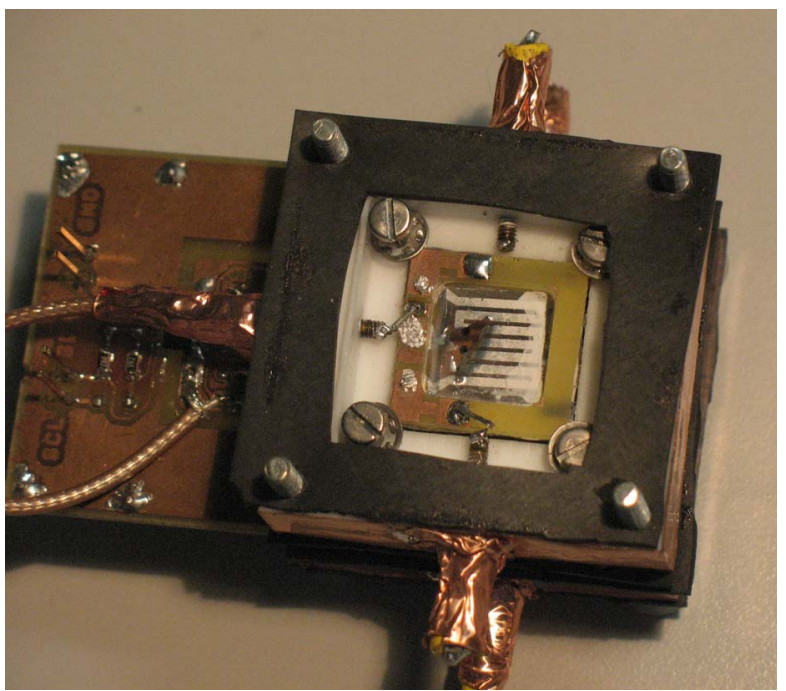

Fig. 2. Picture of the sensors cell including two Mylar membranes with the top opened. The sensor's membrane is cut and the cell medium copper ground plan is seen. White color of the Al meander come from the depositied zeolite film.

\section{1) Mylar membranes}

The first fabrication route used industrial Mylar sheets capped with $30 \mathrm{~nm}$ aluminum films with 2-4 $\Omega_{\square}$ resistance. Mylar sheets of thickness from 2.5 to $22 \mu \mathrm{m}$ were used. A specific procedure to induce a reproducible strain was developed to epoxy glue the Mylar sheets on $1 \mathrm{~cm}$ wide FR4 PCB emptied square. Three gold contacts were deposited on 
the membrane by ion beam sputtering through a mechanical mask. Photolithography and wet etching were then used to pattern a meander shaped aluminum electrode with the three contacts.

\section{2) $\mathrm{Si}_{3} \mathrm{~N}_{4} / \mathrm{SiO}_{2}$ membrane}

The second route used for the MEMS fabrication is to prepare $1 \mathrm{~mm}$ wide $\mathrm{Si}_{3} \mathrm{~N}_{4} / \mathrm{SiO}_{2}$ membrane on $\mathrm{Si}$. $200 \mathrm{~nm}$ Aluminum thin films were deposited on $\mathrm{Si}_{3} \mathrm{~N}_{4} / \mathrm{SiO}_{2}$ capped silicon wafers $(120 \mathrm{~nm} / 1 \mu \mathrm{m}$ respectively). Aluminum $50 \mu \mathrm{m}$ wide meander patterning was realized by standard photolithography and etching. Membranes were released by TMAH (Tetramethylammonium hydroxide) wet etching of the backside silicon. A picture of the membrane is presented Fig. 3.

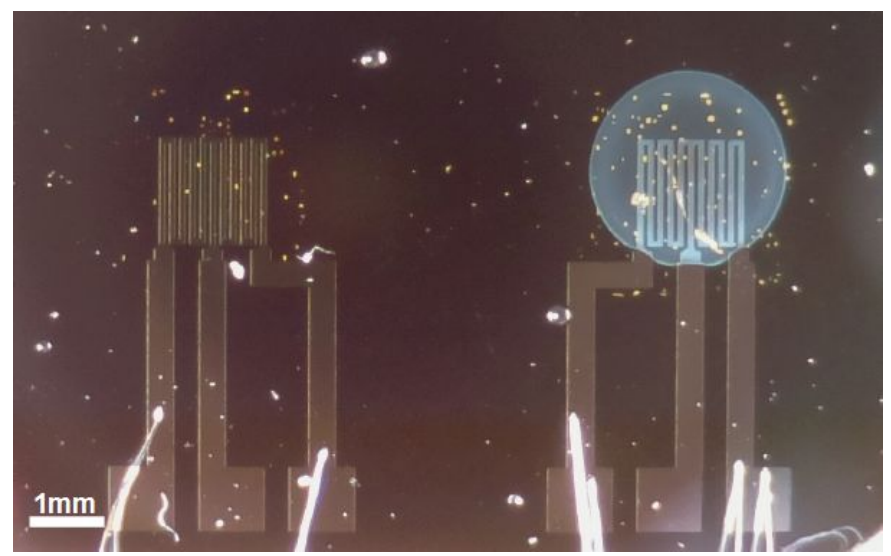

Fig. 3. $\mathrm{Si}_{3} \mathrm{~N}_{4}$ membranes: left is a reference membrane (as prepared) and right is covered with a zeolite film.

While Mylar membranes provide far larger sensible area $\left(\mathrm{cm}^{2}\right.$ vs. $\left.\mathrm{mm}^{2}\right)$, silicon membranes are lighter and use the reproducible mass fabrication procedures of the MEMS technology.

\section{3) Zeolites coating}

In both case, the zeolite film was deposited by drop coating technique. In order to ensure the preparation of smooth and homogenous films, the zeolite crystals with MFI type framework structure were re-dispersed in ethanol (coating suspensions) with different solid contents (1 to $10 \mathrm{wt}$. \%) that controls the films thickness. After drying at ambient condition, the zeolite films were annealed at $80{ }^{\circ} \mathrm{C}$ for 20 minutes. The thickness of the zeolite films was varied by changing the concentration of the zeolite crystals in the coating suspension or by multi-step deposition. Several zeolite films were prepared and thicknesses were confirmed by scanning electron microscope (SEM)

\section{B. Mylar Sensors cell}

The electrical contacts on the aluminum meander on the Mylar membranes, were prepared by pressed indium onto the gold contacts through another $1 \mathrm{~cm}$ wide FR4 PCB emptied square with appropriate copper leads. A picture of the cell with two sensible Mylar membranes is shown Fig. 2 and 4.

The top detector is exposed to the gas mixture while the bottom one is hermetically sealed under an argon atmosphere in order to act as a reference. The two side contacts may be used to heat the meander above $110^{\circ} \mathrm{C}$ in order to desorb the analyte as shown Fig. 4.

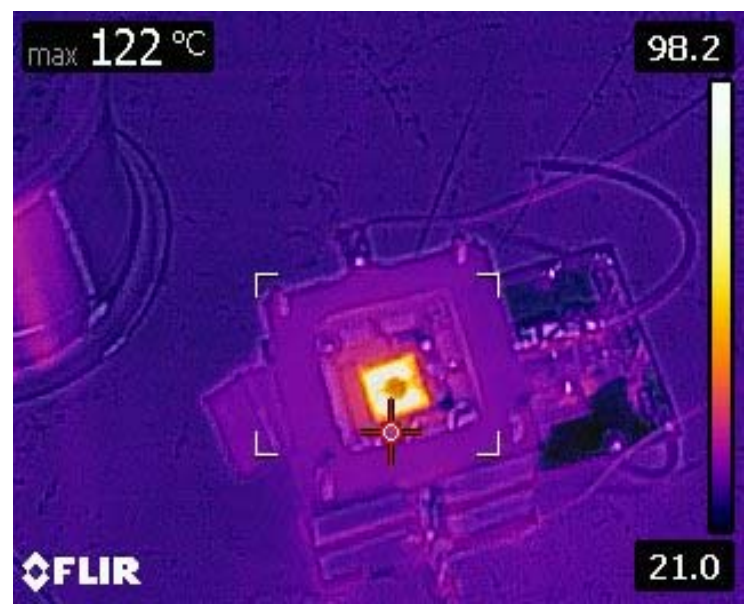

Fig. 4. FLIR image of the detection cell with a $35 \mathrm{~mA}$ heating current through the $30 \mathrm{~nm}$ thick aluminum meander.

\section{The flow system}

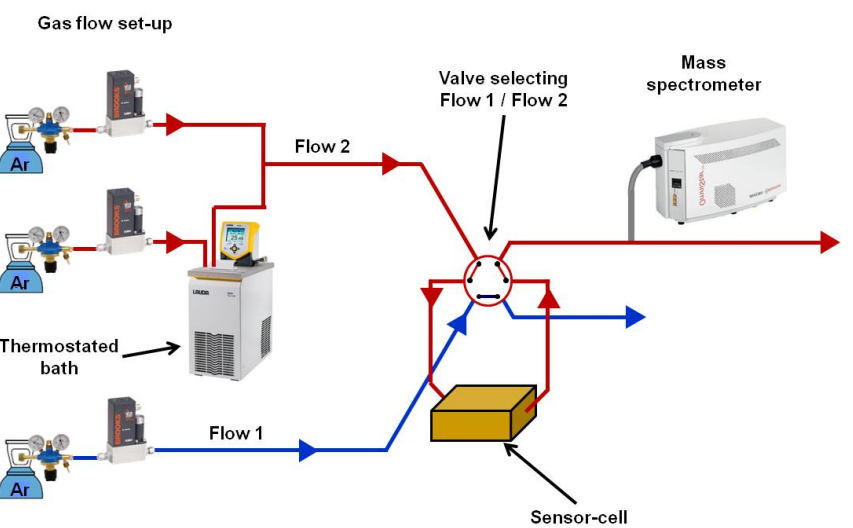

Fig. 5. Schematic general view of the flow system used to deliver analytes with defined concentrations.

The flow system, which was used for this study, is shown in Fig. 5. This system is composed of four main parts: the gas flow set-up, the sensor cell, the flow1/flow2 selection valve and the exhaust gas analyzer. The sensor cell was connected to the gas flow set-up including mass flow controllers for the introduction of gases into the lines. The two gas mixtures (called flow1 and flow2) were prepared and sent independently to the sensor-cell via the flow1/flow2 selection valve (Valco 6 port valve). The system allows investigating the exhaust gases composition (mainly used in this study for the flow2 which contains methanol) by a quadrupole mass spectrometer (Pfeiffer Omnistar GSD 301) with a time-resolution of 1 second. The methanol vapor was sent in the flow2 via a saturator immersed in a thermostated bath purged by an Argon flow. Using the appropriate parameters of the Antoine equation, the required concentration of methanol [0-20 000 $\mathrm{ppm}$ ] is provided accurately by the control of both the temperature of the bath and the velocity of the flow. The 
experiments were performed at a constant temperature $\left(25^{\circ} \mathrm{C}\right)$ with the following flow compositions:

- Flow1 (for the pretreatment): $20 \mathrm{~cm}^{3} \cdot \mathrm{min}^{-1}$ of Ar.

- Flow2: $\mathrm{CH}_{3} \mathrm{OH}$ diluted in Ar for a total flow of 20 $\mathrm{cm}^{3} \cdot \min ^{-1}$

Before the measurements, the sensor-cell was purged under flow 1 at $25^{\circ} \mathrm{C}$.

\section{RESUlTS}

\section{A. Mylar membranes cell}

The presented Mylar membranes cell was exposed to different concentration of methanol while capacitance value of both $\mathrm{Al}$ electrodes with respect to the ground was monitored live. A steady reference signal confirmed the reliable insulation of the bottom detector.

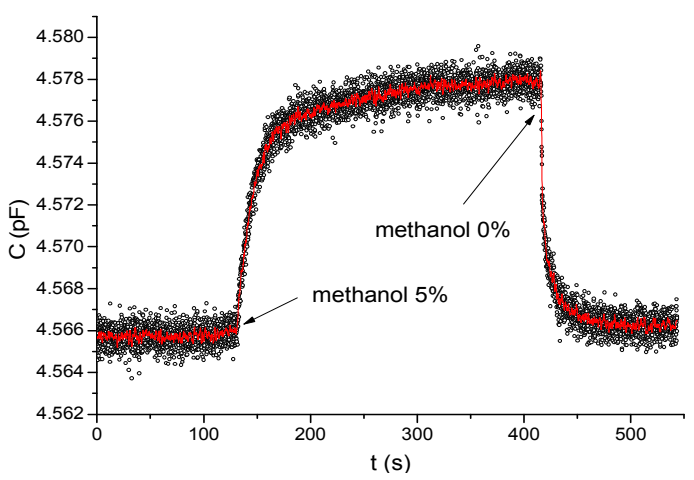

Fig. 6. Top detector capacity vs. time. Dots (black) : experimental data; Line (red) : stavinsky-golay smoothing over $2 \mathrm{~s}$

Fig. 6 presents the top capacitance evolution when exposed to $5 \%$ methanol from $\mathrm{t} \sim 130 \mathrm{~s}$ to $\mathrm{t} \sim 430 \mathrm{~s}$. An increase of 2.3 $\mathrm{fF}$ per $1 \%$ methanol is measured for a noise level around $2 \mathrm{fF}$. We observe a clean return to the initial state. Both responses (on/off) are showing a characteristic time around $20 \mathrm{~s}$. The absorption of methanol in the zeolite pores (rise) keeps a linear component for more than $200 \mathrm{~s}$ while the desorption is purely exponential.

Another experiment was made using a thicker membrane

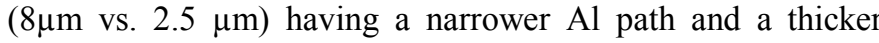
zeolites film $(20-50 \mu \mathrm{m}$ vs. $1 \mu \mathrm{m})$. An enhanced sensitivity to a $0.1 \%$ level of methanol was obtained, while keeping the same noise level. The later, at first glance, is limited by the noise level of the capacitive to digital circuit used (FDC 1004 from $\mathrm{TI}$ ), lying at $40 \mathrm{aF} / \sqrt{\mathrm{Hz}}$ in a noise bandwidth at $1 \mathrm{kHz}$ as given in the datasheet when reading at 400 samples per second.

\section{B. Thermal measurment on silicon membranes}

The calorimetric response of $\mathrm{Si}_{3} \mathrm{~N}_{4} / \mathrm{SiO}_{2}$ membrane sensors was tested in the presence and absence of other analyte, in this case acetone vapor in air. A succession of square shaped thermal spike was applied to the Al meander by Joule effect (Blue signal Fig. 7). The conditioning electronics described in [2] was used to plot a voltage image $\left(\mathrm{V}_{\mathrm{T}}\right.$, red curve Fig. 7) of the sensor temperature variation. On Fig. 7, red up arrows materialize the approach of an acetone vapor source and blue down arrows its retreat. Grey arrows show an approach without acetone with no effect in order to discriminate from potential parasitic effects.

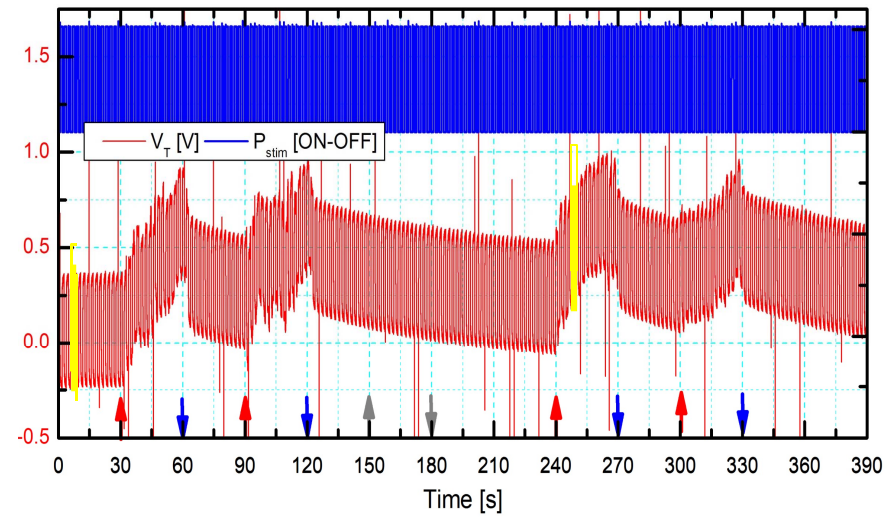

Fig. 7. Thermal response vs. time of the sensor (red, down) to square heating spike (blue, up)

The Two yellow zones were used to measure the thermal time constant of the sensor with or without acetone. A reproducible and significant difference of 5 to $7 \%$ was obtained.

\section{CONCLUSION}

The Single Input - Multi Output (SIMO) general concept is developed based on selective chemical sensing using zeolite layers. Zeolites act as a single proof body to capture the selected chemical species, the adsorption of which produces both a mass and an electrical polarizability variation. These physical quantities then can be evaluated using various excitations and reading mechanisms to obtain the multiple system outputs. Results on capacitive reading of standard metallic, meander like electrodes, have been obtained, while such meanders can also be read using resistive processes together with heating processes needed to get heat capacitance variation associated to the adsorbed mass. The first results demonstrate the pertinence of the SIMO concept in the field of chemical sensing.

\section{REFERENCES}

[1] D. Robbes, G. Allègre, S. Flament, S. Lebargy, A. Swinton and O Masséglia "One Input - Multi Output Sensors: a Relevant Concept", Proceedings of IEEE Sensors 2014, pp. 274-276, November 2-5, 2014.

[2] (a) M. Denoual, G. Allègre, S. Delaunay, D. Robbes, "Capacitively coupled electrical substitution for resistive bolometer enhancement", Meas. Sci. Technol., Vol 20, 015105, 2009; (b) M. Denoual, S Delaunay, D. Robbes, "Bolometer with heat feedback", WO/2009/034066, 2009.

[3] (a) T. Bein, Chem. Mater., 1996, 8, 1636; (b) A. Tavolaro and E. Drioli, Adv. Mater., 1999, 11, 975; (c) P. Varsani, A. Afonja, D. E. Williams, I. P. Parkina and R. Binions, Sensors and Actuators B, 2011, 160, 475.

[4] (a) I. Sasaki, H. Tsuchiya, M. Nishioka, M. Sadakata and T. Okubo, Sensors and Actuators B, 2002, 86, 26; (b) D. J. Wales et al., Chem. Soc. Rev. 2015, 44, 4290-4321. 